Pacific Journal of Mathematics

ON CHARACTERIZATIONS AND INTEGRALS O 


\title{
ON CHARACTERIZATIONS AND INTEGRALS OF GENERALIZED NUMERICAL RANGES
}

\author{
Moshe Goldberg and E. G. Straus
}

Let $c=\left(\gamma_{1}, \cdots, \gamma_{n}\right)$ be given. The generalized numerical range of an $n \times n$ matrix $A$, associated with $c$, is the set $W_{c}(A)=\left\{\Sigma \gamma_{j}\left(A x_{j}, x_{j}\right)\right\}$ where $\left(x_{1}, \cdots, x_{n}\right)$ varies over orthonormal systems in $C^{n}$. Characterizations of this range, for real $c$, are given. Next, we study integrals of the form $\int W_{c}(A) d \mu(c)$ where $\mu(c)$ is a measure defined on a domain in $\boldsymbol{R}^{n}$. The above characterizations are used to study the inclusion $\int W_{\mathrm{c}}(A) d \mu(c) \subset \lambda W_{\mathrm{c}^{\prime}}(A)$. We determine those $\lambda$, for which this inclusion holds for all $n \times n$ matrices $A$. Such relations lead to more elementary ones, when the integral reduces to a finite linear combination of ranges. In particular, we obtain the inclusion relations of the form $W_{c}(A) \subset$ $\lambda W_{c^{\prime}}(A)$ which hold for all $A$.

1. Introduction. The generalized numerical range of an $n \times n$ complex matrix $A$, associated with a fixed vector $c=\left(\gamma_{1}, \cdots, \gamma_{n}\right) \in$ $C^{n}$, is the set of complex numbers

$$
W_{c}=W_{\left(r_{1}, \cdots, r_{n}\right)}(A)=\left\{\sum_{j=1}^{n} \gamma_{j}\left(A x_{j}, x_{j}\right):\left(x_{1}, \cdots, x_{n}\right) \in \Lambda_{n}\right\},
$$

where $A_{n}$ is the set of all orthonormal $n$-tuples of vectors is $C^{n}$. We call $W_{c}$ a generalized range since for $c=(1,0, \cdots, 0)$ it reduces to the classical range

$$
W(A)=\{(A x, x):\|x\|=1\} .
$$

It is clear from (1.1) that $W_{c}$ remains invariant under permutations of the components of $c$; that is, $W_{c}$ depends on the unordered set $\left\{\gamma_{1}, \cdots, \gamma_{n}\right\}$ rather than on $c$.

Westwick, [5], has shown that if $c$ is a real vector then $W_{c}$ is convex, but if $c \in C^{n}$ with $n \geqq 3$, then $W_{c}(x)$ may fail to be convex even for normal $A$. For this reason we restrict our attention, in this paper, to generalized numerical ranges with real coefficients.

Our first purpose is to characterize the sets $W_{c}$. In $\S 2$ we show that

$$
W_{c}(A)=\left\{\operatorname{tr}(H A): H \in \mathscr{H}_{c}\right\},
$$

where $\mathscr{H}_{c}$ is a class of Hermitian matrices depending on $c$.

In $\S 3$ we define integrals of the form $\int_{\mathscr{D}} W_{c}(A) d \mu(c)$ where $\mathscr{D}$ 
is a domain in $\boldsymbol{R}^{n}$ and $\mu(c)$ is a nonnegative measure on $\mathscr{D}$. Since the sets $W_{c}$ are convex, such integrals are convex as well, and we may define them in terms of their support functions.

Finally, using the above characterization of $W_{c}$, we investigate inclusion relations of the form

$$
\int_{\mathscr{D}} W_{c}(A) d \mu(c) \subset \gamma W_{c^{\prime}}(A), \quad \lambda=\text { constant },
$$

which hold, uniformly, for all $A \in \boldsymbol{C}_{n \times n}$, i.e., for all $n$-square matrices. If the measure $\mu(c)$ is concentrated on a finite number of vectors $c$, then (1.2) is reduced to inclusion relations involving finite linear combinations of generalized numerical ranges. Such relations were considered in earlier works [2, 3].

In particular, for given vectors $c, c^{\prime}$ we obtain necessary and sufficient conditions under which

$$
W_{c}(A) \subset \lambda W_{c^{\prime}}, \quad \forall A \in \boldsymbol{C}_{n \times n} .
$$

2. Characterization of generalized ranges. For any vector $c=\left(\gamma_{1}, \cdots, \gamma_{n}\right)$ consider the diagonal matrix

$$
C=\operatorname{diag}(c)=\operatorname{diag}\left(\gamma_{1}, \cdots, \gamma_{n}\right),
$$

and construct the class of matrices

$$
\mathscr{U}_{c}=\operatorname{conv}\left\{U C U^{*}: U \text { unitary }\right\},
$$

where conv denotes the convex hull.

Since we restrict attention to $c \in \boldsymbol{R}^{n}$ it is evident that the elements of $\mathscr{U}_{c}$ are Hermitian.

Using $\mathscr{U}_{c}$ we have the following characterization of ranges with real coefficients.

THEOREM 1. If $c \in \boldsymbol{R}^{n}$ then

$$
W_{c}(A)=\left\{\operatorname{tr}(H A): H \in \mathscr{Q}_{c}\right\} .
$$

Proof. It follows from the definition of $W_{c}(A)$ in (1.1) that

$$
W_{c}(A)=\left\{\operatorname{tr}\left(C U^{*} A U\right): U \text { unitary }\right\} .
$$

Thus

$$
\left.W_{c}(A)=\left\{\operatorname{tr}\left(\left(U C U^{*}\right)\right) A\right): U \text { unitary }\right\},
$$

which implies that

$$
W_{c}(A) \subset\left\{\operatorname{tr}(H A): H \in \mathscr{K}_{c}\right\} .
$$


For the converse inclusion let

$$
H=\sum_{i} \lambda_{i}\left(U_{i} C U_{i}^{*}\right) ; \lambda_{i} \geqq 0, \quad \sum_{i} \lambda_{i}=1,
$$

be an arbitrary element of $\mathscr{U}_{c}$. By the convexity of $W_{c}$ and by (2.1) we have

$$
\operatorname{tr}(H A)=\sum \lambda_{i} \operatorname{tr}\left(\left(U_{i} C U_{i}^{*}\right) A\right) \in W_{c}(A) .
$$

So,

$$
\left\{\operatorname{tr}(H A): H \in \mathscr{U}_{c}\right\} \subset W_{c}(A),
$$

and the theorem follows.

We introduce two definitions which lead to another characterization of $W_{c}(A)$.

Definition 1. (i) A real vector $c=\left(\gamma_{1}, \cdots, \gamma_{n}\right)$ is called ordered if

$$
\gamma_{1} \geqq \gamma_{2} \geqq \cdots \geqq \gamma_{n}
$$

(ii) We say that $c, c^{\prime}$ satisfy $c^{\prime} \prec c$ if there exists a doubly stochastic matrix $S$ (i.e., a matrix with nonnegative entries whose row sums and columns sums equal 1 ), such that $c^{\prime}=S c$.

In Theorem 5 of [3] we proved the following.

Lemma 1. For ordered $c, c^{\prime}$ we have $c^{\prime} \prec c$ if and only if

$$
\sum_{j=1}^{l} \gamma_{j}^{\prime} \leqq \sum_{j=1}^{l} \gamma_{j}, \quad l=1, \cdots, n,
$$

with equality for $l=n$.

Definition 2. Let $c \in \boldsymbol{R}^{n}$, and let $\Lambda_{l}(1 \leqq l \leqq n)$ be the set of all orthonormal $l$-tuples of vectors in $C^{n}$. We define $\mathscr{H}_{0}$ to be the class of all Hermitian matrices $H$ for which

$$
\sum_{j=1}^{l}\left(H x_{j}, x_{j}\right) \leqq \sum_{j=1}^{l} \gamma_{j}, \quad \forall\left(x_{1}, \cdots, x_{l}\right) \in \Lambda_{l}, \quad l=1, \cdots, n,
$$

with equality for $l=n$.

Let $e_{1}, \cdots, e_{n}$ be the standard basis of $\boldsymbol{C}^{n}$. Note that if $\Sigma \gamma_{j}=0$ (which is the case assumed in $\S 3$ ), then the equality for $l=n$ in (2.2) implies that

$$
\sum_{j=1}^{n}\left(H e_{j}, e_{j}\right)=\Sigma \gamma_{j}=0 ;
$$

i.e., all members of $\mathscr{H}_{c}$ have trace 0 . 
Lemma 2. If $c$ is ordered then $\mathscr{H}_{c}=\mathscr{U}_{c}$.

Proof. Take a unitary matrix $U$ and orthonormal vectors $x_{1}, \cdots, x_{l}$, $(1 \leqq l \leqq n)$. Since the vectors $y_{j}=U^{*} x_{j}, j=1, \cdots, l$, are orthonormal as well, it is not hard to verify that

$$
\begin{aligned}
\sum_{j=1}^{l}\left(U C U^{*} x_{j}, x_{j}\right) & =\sum_{j=1}^{l}\left(C y_{j}, y_{j}\right) \leqq \gamma_{1}+\cdots+\gamma_{l}, \\
C & =\operatorname{diag}(c),
\end{aligned}
$$

with equality for $l=n$. Therefore, if

$$
H=\sum_{i} \lambda_{i} U_{i} C U_{i}^{*}, \quad\left(\lambda_{i} \geqq 0, \quad \sum_{i} \lambda_{i}=1\right),
$$

is any (Hermitian) matrix in $\mathscr{U}_{c}$, we find by (2.3) that

$$
\sum_{j=1}^{l}\left(H x_{j}, x_{j}\right)=\sum_{j=1}^{l} \sum_{i} \lambda_{i}\left(U_{i} C U_{i}^{*} x_{j}, x_{j}\right) \leqq \sum_{i} \lambda_{i} \sum_{j=1}^{l} \gamma_{j}=\sum_{j=1}^{l} \gamma_{j},
$$

with equality for $l=n$. So, by Definition $2, H \in \mathscr{H}_{c}$, and consequently $\mathscr{U}_{c} \subset \mathscr{H}_{c}$.

Conversely, take any $H \in \mathscr{H}_{c}$. Since $H$ is Hermitian, it is unitarily similar to a real diagonal matrix, i.e., there exists a unitary $V$ such that

$$
C^{\prime} \equiv V^{*} H V=\operatorname{diag}\left(\gamma_{1}^{\prime}, \cdots, \gamma_{n}^{\prime}\right),
$$

where we may assume that $c^{\prime}=\left(\gamma_{1}^{\prime}, \cdots, \gamma_{n}^{\prime}\right)$ is ordered. Using (2.2) and the orthonormal vectors $x_{j}=V e_{j}, j=1, \cdots, l$, we find that

$$
\sum_{j=1}^{l} \gamma_{j}^{\prime}=\sum_{j=1}^{l}\left(C^{\prime} e_{j}, e_{j}\right)=\sum_{j=1}^{l}\left(V^{*} H V e_{j}, e_{j}\right)=\sum_{j=1}^{l}\left(H x_{j}, x_{j}\right) \leqq \sum_{j=1}^{l} \gamma_{j},
$$

with equality for $l=n$. That is, by Lemma $1, c^{\prime} \prec c$. Hence, there exists a doubly stochastic matrix $S$ such that $c^{\prime}=S c$. Now recall that doubly stochastic matrices are convex combinations of permutation matrices $P_{\sigma}$. In particular $S=\Sigma_{\sigma} \lambda_{\sigma} P_{\sigma}$. Thus

$$
c^{\prime}=\sum_{\sigma \in S_{n}} \lambda_{\sigma} P_{\sigma} c ; \lambda_{\sigma} \geqq 0, \quad \Sigma \lambda_{\sigma}=1,
$$

where $S_{n}$ is the symmetric group. Since for every $B, P_{\sigma} B P_{\sigma}^{*}$ has both the rows and columns of $B$ permuted according to $\sigma$, we have

$$
\operatorname{diag}\left(P_{\sigma} c\right)=P_{\sigma} \operatorname{diag}(c) P_{c}^{*}=P_{\sigma} C P_{\sigma}^{*} .
$$

So, by (2.5), (2.6),

$$
C^{\prime}=\operatorname{diag}\left(c^{\prime}\right)=\sum_{\sigma} \lambda_{\sigma} \operatorname{diag}\left(P_{\sigma} c\right)=\sum_{\sigma} \lambda_{\sigma} P_{\sigma} C P_{\sigma}^{*} .
$$


From (2.4) and (2.7) we obtain

$$
\begin{aligned}
H=V C^{\prime} V^{*}= & \sum_{\sigma} \lambda_{\sigma}\left[\left(V P_{o}\right) C\left(V P_{o}\right)^{*}\right]=\sum_{\sigma} \lambda_{\sigma}\left(U_{\sigma} C U_{\sigma}^{*}\right), \\
& \lambda_{\sigma} \geqq 0, \quad \Sigma \lambda_{\sigma}=1,
\end{aligned}
$$

where $U_{\sigma} \equiv V P_{\sigma}$ are, of course, unitary. Hence, $H \in \mathscr{U}_{c}$, i.e., $\mathscr{H}_{c} \subset \mathscr{U}_{c}$ and the proof is complete.

Theorem 1 together with Lemma 2 imply a second characterization of generalized numerical ranges with real coefficients.

THEOREM 2. If $c$ is ordered then

$$
W_{c}(A)=\left\{\operatorname{tr}(H A): H \in \mathscr{H}_{c}\right\} .
$$

Another simple consequence of the last lemma and the convexity of $\mathscr{U}_{c}$ is that for ordered $c, \mathscr{H}_{c}$ is convex.

At this point we recall the definition of the $k$-numerical range, $(1 \leqq k \leqq n)$, given by Halmos [1, 1167$]$, which after a convenient normalization becomes

$$
W_{k}(A)=\left\{\frac{1}{k} \operatorname{tr}(P A P): P=\text { orthogonal projection of rank } k\right\} .
$$

It can be verified that $W_{k}(A)$ may be written as

$$
W_{k}(A)=\left\{\frac{1}{k} \sum_{j=1}^{k}\left(A x_{j}, x_{j}\right):\left(x_{1}, \cdots, x_{k}\right) \in \Lambda_{k}\right\} .
$$

Hence we see that

$$
W_{k}(A)=W_{c_{k}}(A), \quad \text { with } c_{k}=\frac{1}{k}\left(e_{1}+\cdots+e_{k}\right) .
$$

That is, the $k$-numerical range is a special case of the generalized numerical range.

The matrices $\mathscr{H}_{c_{k}}$ are those Hermitian matrices which satisfy Definition 2 with $c=c_{k}$. Using this definition one can show that

$$
\mathscr{H}_{c_{k}}=\left\{\text { Hermitian } H: 0 \leqq H \leqq \frac{1}{k} I, \operatorname{tr}(H)=1\right\} \cdot
$$

Thus Theorem 2 generalizes the result

$$
W_{k}(A)=\left\{\operatorname{tr}(H A): 0 \leqq H \leqq \frac{1}{k} I, \operatorname{tr}(H)=1\right\}, k=1, \cdots, n
$$

of Fillmore and Williams [1, Theorem 1.2].

3. Integrals of generalized ranges. In this section we are 
interested in linear combinations, or more generally, in integrals of the sets $W_{c}(A)$, where $A$ is arbitrary but fixed, and $c$ varies in some domain of $\boldsymbol{R}^{n}$.

Let $c=\left(\gamma_{1}, \cdots, \gamma_{n}\right)$ be a real vector with $\gamma \equiv \Sigma \gamma_{j} \neq 0$, and consider the vector $b=\left(\beta_{1}, \cdots, \beta_{n}\right)$ defined by

$$
b=c-\left(\frac{\gamma}{n}, \cdots, \frac{\gamma}{n}\right) .
$$

We have $\Sigma \beta_{j}=0$ and

$$
B \equiv \operatorname{diag}(b)=\operatorname{diag}(c)-\frac{\gamma}{n} I=C-\frac{\gamma}{n} \dot{I} .
$$

So, by Theorem 1,

$$
\begin{aligned}
& W_{b}(A)=\left\{\operatorname{tr}\left(U B U^{*} A\right): U \text { unitary }\right\} \\
& \quad=\left\{\operatorname{tr}\left[U\left(C-\frac{\gamma}{n} I\right) U^{*} A\right]: U \text { unitary }\right\}=W_{c}(A)-\left\{\frac{\gamma}{n} \operatorname{tr}(A)\right\} .
\end{aligned}
$$

This argument suggests that it is convenient to restrict attention to those vectors $c$ for which $\Sigma \gamma_{j}=0$. The limitation merely involves a translation of the ranges by multiples of the trace, or, equivalent$l y$, the restriction to matrices of trace 0 .

Since $W_{c}$ is invariant under permutations of the $\gamma_{j}$, we may assume that each vector $c$ in our domain is ordered. Hence, we consider the set of ordered vectors $c$ with $\Sigma \gamma_{j}=0$, which form a conical subset $\mathscr{C}$ of an $(n-1)$-dimensional subspace of $\boldsymbol{R}^{n}$.

We are ready now to study integrals of $W_{c}(A)$ relative to an arbitrary measure $\mu$ on $\mathscr{C}$, that is integrals of the form

$$
J_{\mu}=J_{\mu}(A)=\int_{\varnothing} W_{c}(A) d \mu(c) .
$$

One way of defining the integral in (3.1) is by carrying linear sums, over partitions of $\mathscr{C}$, to the limit. Alternatively, one realizes that $J_{\mu}$, being an integral of the convex sets $W_{c}$, is a convex set as well. Hence $J_{\mu}$ may be characterized by its support function (e.g., [4] part V),

$$
u\left(J_{\mu}, \theta\right)=\sup _{z \in J_{\mu}} \operatorname{Re}\left(z e^{-i \theta}\right), \quad 0 \leqq \theta<\pi
$$

In order to evaluate $u\left(J_{\mu}, \theta\right)$, we consider the support functions of our closed convex integrands $W_{c}$. We have

$$
u\left(W_{c}, \theta\right)=u(c, \theta)=\max _{z \in W_{c}} \operatorname{Re}\left(z e^{-i \theta}\right), \quad 0 \leqq \theta<\pi .
$$

Since $u(c, \theta)$ is a linear function of $c$ in the sense that 


$$
u\left(\lambda W_{c}+\lambda^{\prime} W_{c^{\prime}}, \theta\right)=\lambda u(c, \theta)+\lambda^{\prime} u\left(c^{\prime}, \theta\right), \quad \forall \lambda, \lambda^{\prime} \geqq 0,
$$

we have

$$
u\left(J_{\mu}, \theta\right)=u\left(\int W_{c} d \mu(c), \theta\right)=\int u\left(W_{c}, \theta\right) d \mu(c)=\int u(c, \theta) d \mu(c) .
$$

Of course, the measure $\mu$ may be concentrated at a finite number of points $c_{1}, \cdots, c_{m} \in \mathscr{C}$. In this case the integral $J_{\mu}$ reduces to the finite linear combination

$$
\mu\left(c_{1}\right) W_{c_{1}}(A)+\cdots+\mu\left(c_{m}\right) W_{c_{m}}(A) .
$$

Since $W_{\lambda c}=\lambda W_{c}$ for scalar $\lambda$, we shall avoid integration over proportional vectors of $\mathscr{C}$. This can be achieved by restricting integration to the domain

$$
\mathscr{D}=\left\{c: c=\left(\gamma_{1}, \cdots, \gamma_{n}\right), \Sigma \gamma_{j}=0, \quad \gamma_{1}=1\right\},
$$

which is the bounded set of all vectors in $\mathscr{C}$ with $\gamma_{1}=1$.

The above concept of integration can be extended in order to consider the integral

$$
\mathscr{H}_{\mu} \equiv \int_{\sim} \mathscr{H}_{c} d \mu(c)
$$

We recall that the integrands $\mathscr{H}_{c}$ are convex sets in the $\left(n^{2}-1\right.$ real dimensional) space $\boldsymbol{H}$ of Hermitian matrices of trace 0 . It follows that $\mathscr{H}_{\mu}$ is also a convex set in $\boldsymbol{H}$. Again, the convexity of $\mathscr{H}_{c}$ and $\mathscr{H}_{\mu}$ implies that the integral may be defined in terms of the support functions of $\mathscr{H}_{c}$. Here, in analogy to the previous case, the support function of $\mathscr{H}_{c}$ assigns to each point $H_{1}$ on the unit sphere of $\boldsymbol{H}$, the distance from the origin $O$ of $\boldsymbol{H}$ to the plane of support of $\mathscr{H}_{c}$ perpendicular to the direction $\overrightarrow{O H}_{1}$.

Having the integrals $J_{\mu}$ and $\mathscr{H}_{\mu}$ defined we state our main result.

THEOREM 3. Let $\mu$ be a nonnegative measure on $\mathscr{D}$, and let $c^{\prime} \neq 0$ be an ordered vector with $\Sigma \gamma_{j}^{\prime}=0$. Then

$$
\int_{\mathscr{\partial}} W_{c}(A) d \mu(c) \subset \lambda W_{c^{\prime}}(A), \quad \forall A \in \boldsymbol{C}_{n \times n},
$$

if and only if $\lambda \geqq \eta\left(c^{\prime}\right)$ or $\lambda \leqq \zeta\left(c^{\prime}\right)$ where

$$
\begin{aligned}
& \eta\left(c^{\prime}\right)=\max _{1 \leq l<n} \int_{\mathscr{D}} \frac{\gamma_{1}+\cdots+\gamma_{l}}{\gamma_{1}^{\prime}+\cdots+\gamma_{l}^{\prime}} d \mu(c), \\
& \zeta\left(c^{\prime}\right)=\min _{1 \leq l<n} \int_{-} \frac{\gamma_{1}+\cdots+\gamma_{l}}{\gamma_{n}^{\prime}+\cdots+\gamma_{n-l+1}^{\prime}} d \mu(c) .
\end{aligned}
$$


Proof. In the proof of Lemma 8 of [3] we have shown that if $c^{\prime} \neq 0$ with $\Sigma \gamma_{j}^{\prime}=0$, then

$$
\gamma_{1}^{\prime}+\cdots+\gamma_{l}^{\prime}>0, \quad \gamma_{n}^{\prime}+\cdots+\gamma_{n-l+1}^{\prime}<0 ; l=1, \cdots, n-1 .
$$

This establishes that $\eta, \zeta$ of (3.4) are well defined and since $\mu$ is a nonnegative measure we see that $\eta \geqq 0, \zeta \leqq 0$.

Next we show that $\lambda \geqq \eta\left(c^{\prime}\right)$ or $\lambda \leqq \zeta\left(c^{\prime}\right)$ imply (3.3). For this purpose we use the definition of $\mathscr{H}_{\mu}$, Theorem 2, and the linearity of the trace to evaluate the set on the left of (3.3):

$$
\begin{aligned}
\int_{\mathscr{D}} W_{c}(A) d \mu(c)=\int_{\mathscr{D}}\left\{\operatorname{tr}(H A): H \in \mathscr{H}_{c}\right\} d \mu(c) \\
=\left\{\operatorname{tr}(H A): H \in \int_{\mathscr{D}} \mathscr{H}_{c} d \mu(c)\right\}=\left\{\operatorname{tr}(H A): H \in \mathscr{H}_{\mu}\right\} .
\end{aligned}
$$

Now choose $\lambda$ with $\lambda \geqq \eta\left(c^{\prime}\right)$. Since $\lambda \geqq 0$, the vector $\lambda c^{\prime}$ remains ordered. Hence, by Theorem 2,

$$
\lambda W_{c^{\prime}}(A)=W_{\lambda . c^{\prime}}(A)=\left\{\operatorname{tr}(H A): H \in \mathscr{H}_{\lambda c^{\prime}}\right\} .
$$

From (3.6), (3.7) we see that in order to prove (3.3) it suffices to show that

$$
\mathscr{H}_{\mu} \subset \mathscr{H}_{\lambda c^{\prime}} .
$$

Thus, let $H_{0}$ be a matrix in $\mathscr{H}_{\mu}$. Then by (3.2), there exist elements $H_{c} \in \mathscr{H}_{c}$ for all $c \in \mathscr{D}$, such that

$$
H_{0}=\int_{\mathscr{D}} H_{c} d \mu^{\prime}(c) \text {. }
$$

The matrices $H_{c}$ satisfy Definition 2 , and since $\mu$ is a nonnegative measure on $\mathscr{D}$, it follows that for $l$-tuples $x_{1}, \cdots, x_{l}$ in $\Lambda_{k}$ we have

$$
\begin{aligned}
& \sum_{j=1}^{l}\left(H_{0} x_{j}, x_{j}\right)=\int_{\mathscr{D}} \sum_{j=1}^{l}\left(H_{\mathrm{c}} x_{j}, x_{j}\right) d \mu(c) \\
& \quad \leqq \int_{\mathscr{D}}\left(\gamma_{1}+\cdots+\gamma_{l}\right) d \mu(c) ; l=1, \cdots, n,
\end{aligned}
$$

with equality for $l=n$. Since $\Sigma \gamma_{j}=\Sigma \gamma_{j}^{\prime}=0$, the above equality for $l=n$ implies

$$
\sum_{j=1}^{n}\left(H_{0} x_{j}, x_{j}\right)=0=\lambda \sum_{j=1}^{n} \gamma_{j}^{\prime} \text {. }
$$

For $1 \leqq l<n$ we use the assumption $\lambda \geqq \eta$ to obtain from (3.9) that 
(3.10b) $\sum_{j=1}^{l}\left(H_{0} x_{j}, x_{j}\right)$

$$
\leqq\left(\gamma_{1}^{\prime}+\cdots+\gamma_{l}^{\prime}\right) \int_{\mathscr{D}} \frac{\gamma_{1}+\cdots+\gamma_{l}}{\gamma_{1}^{\prime}+\cdots+\gamma_{l}^{\prime}} d \mu(c) \leqq \lambda\left(\gamma_{1}^{\prime}+\cdots+\gamma_{l}^{\prime}\right) .
$$

By Definition 2, the relations (3.10) mean that $H_{0} \in \mathscr{H}_{\lambda c^{\prime}}$. Hence, (3.8) holds, and consequently the inclusion in (3.3) follows.

For $\lambda \leqq \zeta$ the situation is slightly different. Consider the vector $c^{\prime \prime} \equiv\left(-\gamma_{n}^{\prime}, \cdots,-\gamma_{1}^{\prime}\right)$. Since $c^{\prime}$ is ordered, $c^{\prime \prime}$ is too. Also, the condition $\lambda \leqq \zeta\left(c^{\prime}\right)$ becomes

$$
\begin{aligned}
-\lambda & \geqq-\zeta\left(c^{\prime}\right)=-\min _{1 \leqq l<n} \int_{\mathscr{D}} \frac{\gamma_{1}+\cdots+\gamma_{l}}{\gamma_{n}^{\prime}+\cdots+\gamma_{n-l+1}^{\prime}} d \mu(c) \\
& =\max _{1 \leqq l<n} \int_{\mathscr{D}} \frac{\gamma_{1}+\cdots+\gamma_{l}}{-\gamma^{\prime}-\cdots-\gamma_{n-l+1}^{\prime}} d \mu(c)=\eta\left(c^{\prime \prime}\right) .
\end{aligned}
$$

Hence, by the previous part of the proof, we have that

$$
\int_{\mathscr{D}} W_{c}(A) d \mu(c) \subset-\lambda W_{c^{\prime \prime}}(A), \quad \forall A \in C_{n \times n} .
$$

But $-\lambda c^{\prime \prime}$ is merely a reordering of $\lambda c^{\prime}$. Thus, the set on the right of (3.12) satisfies

$$
-\lambda W_{c^{\prime \prime}}(A)=W_{-\lambda c^{\prime \prime}}(A)=W_{\lambda c^{\prime}}(A)=\lambda W_{c^{\prime}}(A),
$$

and we obtain (3.3).

To complete the proof we have to show that if $\zeta<\lambda<\eta$, then (3.3) does not hold for some $A \in C_{n \times n}$. First assume $0 \leqq \lambda<\eta$. That is, for some $l, 1 \leqq l<n$,

$$
\lambda\left(\gamma_{1}^{\prime}+\cdots+\gamma_{l}^{\prime}\right)<\int_{\mathscr{D}}\left(\gamma_{1}+\cdots+\gamma_{l}\right) d \mu(c) .
$$

Consider the matrix $A_{l}=I_{l} \oplus O_{n-l}$. A simple computation shows that for an ordered vector $c$, the range $W_{c}\left(A_{l}\right)$ is a real interval with right end-point $\gamma_{1}+\cdots+\gamma_{l}$. Then, the left side of (3.3) represents a real interval with right end-point

$$
\int_{\mathscr{D}}\left(\gamma_{1}+\cdots+\gamma_{l}\right) d \mu(c),
$$

which, by (3.13), exceeds the right end-point $\lambda\left(\gamma_{1}^{\prime}+\cdots+\gamma_{l}^{\prime}\right)$ of $W_{\lambda c^{\prime}}\left(A_{l}\right)$.

Finally, if $\zeta\left(c^{\prime}\right)<\lambda<0$, then (3.11) implies that $0<-\lambda<\eta\left(c^{\prime \prime}\right)$ where $c^{\prime \prime}=\left(-\gamma_{n}^{\prime} \cdots,-\gamma_{1}^{\prime}\right)$. Thus by the above example the inclusion

$$
\int_{\mathscr{D}} W_{c}\left(A_{l}\right) d \mu(c) \subset-\lambda W_{c^{\prime \prime}}\left(A_{l}\right)=\lambda W_{c^{\prime}}\left(A_{l}\right)
$$


fails to hold, and the theorem follows.

We remember of course, that we restricted integration to the domain $\mathscr{D}$ for convenience only. Therefore, if so desired, $\mu(c)$ can be extended to the domain $\mathscr{C}$, and Theorem 3 remains valid.

If $\mu$ is concentrated at a finite number of vectors $c_{1}, \cdots, c_{m} \in \mathscr{C}$, then Theorem 3 characterizes all $\lambda$ for which

$$
\sum_{i=1}^{m} \mu\left(c_{i}\right) W_{c_{i}}(A) \subset \lambda W_{c^{\prime}}(A), \quad \forall A \in \boldsymbol{C}_{n \times n} .
$$

A result of this type is given in Theorem 1 of [2].

Of particular interest is the case where $\mu$ is concentrated at a single vector $c^{\prime \prime} \in \mathscr{C}$. That is,

$$
\int_{\mathscr{D}} W_{c}(A) d \mu(c)=W_{c^{\prime \prime}}(A)
$$

and $\eta, \zeta$ of (3.13) are given now by

$$
\eta\left(c^{\prime}\right)=\max _{1 \leqq l<n} \frac{\gamma_{1}^{\prime \prime}+\cdots+\gamma_{1}^{\prime \prime}}{\gamma_{1}^{\prime}+\cdots+\gamma_{l}^{\prime}} ; \zeta\left(c^{\prime}\right)=\min _{1 \leqq l<n} \frac{\gamma_{1}^{\prime \prime}+\cdots+\gamma_{l}^{\prime \prime}}{\gamma_{n}^{\prime}+\cdots+\gamma_{n-l+1}^{\prime}} .
$$

Thus, from Theorem 3 we conclude,

CoRollary. Let $c^{\prime} \neq 0$ and $c^{\prime \prime}$ be ordered vectors with $\Sigma \gamma_{j}^{\prime}=$ $\Sigma \gamma_{j}^{\prime \prime}=0$. Then

$$
W_{c^{\prime \prime}}(A) \subset \lambda W_{c^{\prime}}(A), \quad \forall A \in C_{n \times n}
$$

if and only if $\lambda \geqq \eta\left(c^{\prime}\right)$ or $\lambda \leqq \zeta\left(c^{\prime}\right)$ where $\eta$, $\zeta$ are given in (3.14).

This result was proved differently in Theorem 8 of [3].

\section{REFERENCES}

1. P. A. Fillmore and J. P. Williams, Some convexity theorems for matrices, Glasgow Math. J., 12 (1971), 110-117.

2. M. Goldberg and E. G. Straus, Inclusion relations involving k-numerical ranges, Linear Algebra and Appl., 15 (1976), 261-270.

3. - Elementary inclusion relations for generalized numerical ranges, Linear Algebra and Appl., 18, No. 1 (1977), to appear.

4. F. A. Valentine, Convex Sets, McGraw-Hill Co., New York, 1964.

5. R. Wertwick, A theorem on numerical range, Linear and Multilinear Algebra, 2 (1975), 311-315.

Received August 2, 1976, and in revised form October 29, 1976. The research of the first author was supported in part by the Air Force Office of Scientific Research, Air Force Systems Command, USAF, under Grant No. AFOSR-76-3046. The work of the second author was sponsored in part by NSF Grant MPS 71-2884. 


\section{PACIFIC JOURNAL OF MATHEMATICS}

EDITORS

RICHARD ARENS (Managing Editor)

University of California

Los Angeles, California 90024

R. A. Beaumont

University of Washington

Seattle, Washington 98105
J. DUGUNDJI

Department of Mathematics

University of Southern Californı

Los Angeles, California 90007

D. Gilbarg and J. Milgram

Stanford University

Stanford, California 94305

\section{ASSOCIATE EDITORS}

E. F. BECKENBACH

B. H. NeumanN

F. WOLF

K. YoSHIDA

\section{SUPPORTING INSTITUTIONS}

UNIVERSITY OF BRITISH COLUMBIA

CALIFORNIA INSTITUTE OF TECHNOLOGY

UNIVERSITY OF CALIFORNIA

MONTANA STATE UNIVERSITY

UNIVERSITY OF NEVADA

NEW MEXICO STATE UNIVERSITY

OREGON STATE UNIVERSITY

UNIVERSITY OF OREGON

OSAKA UNIVERSITY
UNIVERSITY OF SOUTHERN CALIFORNIA

STANFORD UNIVERSITY

UNIVERSITY OF TOKYO

UNIVERSITY OF UTAH

WASHINGTON STATE UNIVERSITY

UNIVERSITY OF WASHINGTON

AMERICAN MATHEMATICAL SOCIETY

NAVAL WEAPONS CENTER 


\section{Pacific Journal of Mathematics}

Vol. 69 , No. 1

May, 1977

V. V. Anh and P. D. Tuan, On starlikeness and convexity of certain analytic

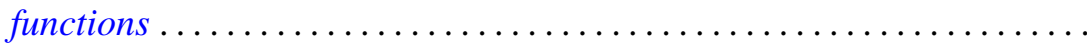

Willard Ellis Baxter and L. A. Casciotti, Rings with involution and the prime

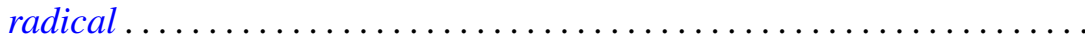

Manuel Phillip Berriozabal, Hon-Fei Lai and Dix Hayes Pettey,

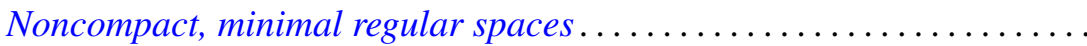

Sun Man Chang, Measures with continuous image law ................

John Benjamin Friedlander, Certain hypotheses concerning

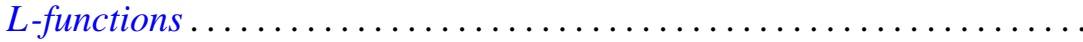

Moshe Goldberg and Ernst Gabor Straus, On characterizations and

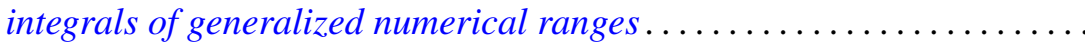

Pierre A. Grillet, On subdirectly irreducible commutative semigroups ...... 55

Robert E. Hartwig and Jiang Luh, On finite regular rings ..............

Roger Hugh Hunter, Fred Richman and Elbert A. Walker, Finite direct sums of cyclic valuated p-groups ........................... 97

Atsushi Inoue, On a class of unbounded operator algebras. III ......... 105

Wells Johnson and Kevin J. Mitchell, Symmetries for sums of the Legendre

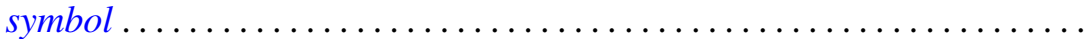

Jimmie Don Lawson, John Robie Liukkonen and Michael William Mislove,

Measure algebras of semilattices with finite breadth

Glenn Richard Luecke, A note on spectral continuity and on spectral properties of essentially $G_{1}$ operators ...............

Takahiko Nakazi, Invariant subspaces of weak-* Dirichlet algebras . .

James William Pendergrass, Calculations of the Schur group ...

Carl Pomerance, On composite $n$ for which $\varphi(n) \mid n-1$. II. . .

Marc Aristide Rieffel and Alfons Van Daele, A bounded operator approach to Tomita-Takesaki theory........................

Daniel Byron Shapiro, Spaces of similarities. IV. $(s, t)$-families ...

Leon M. Simon, Equations of mean curvature type in 2 independent variables.

Joseph Nicholas Simone, Metric components of continuous images of ordered compacta ............................

William Charles Waterhouse, Pairs of symmetric bilinear forms in

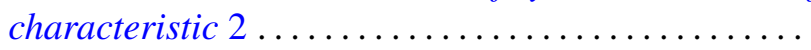

